\title{
Associação entre transtornos mentais comuns e condições subjetivas de saúde entre idosos
}

\author{
The association between common mental disorders \\ and subjective health conditions among the elderly
}

Andréa Maria Eleutério de Barros Lima Martins ${ }^{1}$

Jairo Evangelista Nascimento ${ }^{1}$

João Gabriel Silva Souza ${ }^{2}$

Maria Aparecida Barbosa de Sá ${ }^{1}$

Sara de Barros Lima Feres ${ }^{3}$

Bruno Porto Soares ${ }^{4}$

Efigenia Ferreira e Ferreira ${ }^{5}$

\footnotetext{
${ }^{1}$ Departamento de Odontologia, Centro de Ciências Básicas e da Saúde, Universidade Estadual de Montes Claros. Av. Dr. Rui Braga s/n, Vila Mauriceia. 39401-089 Montes Claros MG Brasil. martins.andreamebl@ gmail.com

${ }^{2}$ Faculdade de Odontologia de Piracicaba, Universidade Estadual de Campinas. Piracicaba SP Brasil.

${ }^{3}$ Instituto de Ciências da Saúde, Associação Educativa do Brasil. Montes Claros MG Brasil.

${ }^{4}$ Faculdades Unidas do Norte. Montes Claros MG Brasil.

${ }^{5}$ Odontologia Social e Preventiva, Universidade Federal de Minas Gerais. Belo Horizonte MG Brasil.
}

Abstract The scope of this study was to evaluate the association between the presence of common mental disorders and the impairment of subjective health conditions among the elderly. It involved a cross-sectional analytical survey conducted among all the elderly residents in the urban area of a Brazilian municipality with low population density. Mental disorders were evaluated using the short version of the Goldberg General Health questionnaire. Subjective and normative health conditions were evaluated and logistic regression was applied (OR/CI95\%) with a 5\% significance level. The survey included 419 elderly residents and the prevalence of mental disorders was $44.6 \%$. The presence of mental disorders was greater among seniors who reported dissatisfaction with life, impairment in the mental and physical control of quality of life and with self-perception of the appearance of teeth and gums as negative. The prevalence of disorders was less identified between men and among those for whom oral health did not affect their relationships with other people The common mental disorder was identified in a significant number of the elderly investigated and the presence of this disorder has been mainly associated with oral health conditions.

Key words Mental health, Elderly, Health, Prevalence, Epidemiology
Resumo Objetivou-se avaliar a associação entre a presença de transtornos mentais comuns e o comprometimento das condições subjetivas de saúde entre idosos. Estudo transversal analitico conduzido entre todos os idosos residentes na área urbana de um município brasileiro de pequeno porte populacional. Os transtornos foram avaliados pela versão abreviada do Questionário de Saúde Geral de Goldberg. Avaliaram-se condições subjetivas e normativas de saúde. Utilizou-se regressão logística (OR/IC95\%) com nível de significância de 5\%. Foram incluídos 419 idosos. A prevalência dos transtornos foi de 44,6\%. A presença de transtornos foi maior entre os idosos que relataram insatisfação com a vida, comprometimento no domínio mental e físico da qualidade de vida e que autoperceberam a aparência de dentes e gengivas como negativa. Menor chance da presença de transtornos foi identificada entre homens e entre aqueles em que a saúde bucal não afetou o relacionamento com outras pessoas. O transtorno mental comum foi identificado em uma parcela considerável dos idosos investigados sendo associado, principalmente, a condições subjetivas de saúde.

Palavras-chave Saúde mental, Idoso, Saúde, Prevalência, Epidemiologia 


\section{Introdução}

O envelhecimento populacional é um dos fenômenos demográficos mais notórios da atualidade. Nos países desenvolvidos, a mudança na estrutura etária da população foi um processo lento e já consolidado. Já naqueles em desenvolvimento, esse processo iniciou-se mais tardiamente e vem ocorrendo num ritmo acelerado ${ }^{1,2}$. Apesar do prolongamento da vida ser uma conquista da sociedade, tal realidade é preocupante no Brasil, onde o envelhecimento populacional pode representar um problema, uma vez que esse processo nem sempre é acompanhado por ampliação da cobertura dos sistemas de proteção social e por melhoria das condições de vida e qualidade de vida das pessoas ${ }^{3}$.

O envelhecimento, processo natural e gradual, produz limitações e alterações no funcionamento do organismo do idoso, com consequente diminuição da qualidade de vida, que pode ser compreendida como um conjunto harmonioso de satisfações que a pessoa obtém no seu cotidiano, levando-se em consideração tanto os aspectos físicos quanto o psicológico e o social ${ }^{4}$. Tem-se observado o aumento da prevalência de enfermidades características deste estrato etário ${ }^{2}$ e dentre os problemas de saúde que acometem os idosos, encontram-se os transtornos mentais. Eles afetam de 20 a $25 \%$ de todas as pessoas em um dado momento de sua vida ${ }^{5}$ e estão presentes em cerca de um terço da população com mais de 60 anos de idade, apesar disso ainda são poucos os estudos epidemiológicos de morbidade psiquiátrica geral no idoso ${ }^{6-8}$. Dentre esses transtornos destacam-se o Transtorno Mental Comum (TMC).

O TMC é considerado uma situação de saúde que não preenche os critérios formais para diagnóstico de depressão e/ou ansiedade segundo as classificações Diagnostic and Statistical Manual of Mental Disorders Fourth Edition (DSM-IV) e da Classificação Internacional de Doenças 10a revisão (CID-10), mas que apresentam sintomas proeminentes que trazem uma incapacitação funcional comparável ou até pior do que quadros crônicos bem estabelecidos ${ }^{9}$. O TMC também denominado distúrbio psiquiátrico menor, transtorno não psicótico e morbidade psiquiátrica menor, se caracteriza por: insônia, fadiga, irritabilidade, esquecimento, dificuldade de concentração, queixas somáticas, episódio de humor, transtorno de humor, abuso de substâncias, ansiedade, transtorno nos hábitos alimentares ${ }^{10}$. Portadores de transtornos psíquicos, em geral, deixam de desempenhar adequadamente o autocuidado, comprometendo, por exemplo, sua saúde ${ }^{11}$.

Estudos prévios têm identificado a associação e o impacto dos TMC com outras condições de saúde, principalmente aquelas de caráter normativo $^{7,8}$. No entanto, sabe-se que as condições normativas por si só não indicam o quanto as pessoas se sentem afetadas pela sua condição de saúde $^{12,13}$. Constata-se ainda serem escassos os estudos populacionais domiciliares que investigam a associação entre TMC e condições subjetivas de saúde, entre elas incluem-se as condições de saúde bucal, situação pouco explorada entre os acometidos por esta enfermidade. Dessa forma, o presente estudo objetivou estimar a prevalência do TMC entre idosos residentes em um município brasileiro de pequeno porte populacional, assim como, verificar se a presença de tal transtorno foi associada ao comprometimento de condições subjetivas de saúde.

\section{Metodologia}

Estudo transversal analítico censitário conduzido entre todos os idosos ( $\geq 60$ anos de idade) residentes e domiciliados em área urbana de um município brasileiro de pequeno porte populacional (Ibiaí - Minas Gerais). Os dados foram coletados, entre janeiro de 2011 a julho 2012, por acadêmicos do décimo período do curso de odontologia segundo padrões estabelecidos pela Organização Mundial de Saúde (OMS), em $1997^{14}$. Participaram da coleta de dados examinadores que foram treinados e calibrados (Kappa simples inter/intra examinadores) em um estudo piloto conduzido entre sessenta idosos de outro município. Os examinadores apresentaram concordância $\geq 0,61$ para todas as condições normativas de saúde bucal avaliadas, conforme escala proposta por Fleiss ${ }^{15}$ e modificada por Cicchetti et al. ${ }^{16}$. Foi aplicado um questionário semiestruturado, precedido pela assinatura do termo de consentimento livre e esclarecido, sendo garantida a privacidade dos participantes durante a coleta de dados. Foram avaliados todos os idosos encontrados após visita a todos os domicílios da área urbana do município e incluídos no presente estudo aqueles que responderam as questões referentes à presença de TMC.

A variável dependente - transtornos mentais comuns - foi avaliada pela versão abreviada do Questionário de Saúde Geral de Goldberg $(\mathrm{QSG})^{17}$, adaptado para a língua portuguesa e cultura brasileira $^{18}$. Trata-se de um instrumen- 
to estruturado que tem por objetivo identificar o perfil sintomático de saúde mental de pessoas com distúrbios psiquiátricos não extremos - transtornos depressivos e de ansiedade de gravidade leve a moderada ${ }^{17}$ considerando cinco fatores: estresse psíquico; desejo de morte; desconfiança do próprio desempenho; distúrbios do sono e distúrbios psicossomáticos, além de escore relativo à saúde geral ${ }^{19}$. $\mathrm{O}$ instrumento contém 12 questões cujas respostas são emitidas numa escala que varia de 1 a 4 pontos, que se referem ao nível de concordância de cada um dos itens apresentados. A apuração das respostas neste estudo seguiu o sistema de pontuação adotado por Goldberg $^{17}$, o qual implica que as respostas um e dois sejam recodificados como "zero" referente à ausência de distúrbio psiquiátrico e as respostas três e quatro sejam recodificados como "um" referente à presença de distúrbio psiquiátrico. Em um segundo momento é feita a soma das doze respostas recodificadas em um e zero gerando o escore do QSG. Os escores gerados variam de um a doze e o ponto de corte é 3/4, ou seja, os idosos que apresentaram escores um, dois e três foram considerados sem transtorno, assim como os que apresentaram o escore zero (ausência de transtorno em todas as questões), e aqueles que apresentaram de quatro a doze foram considerados com transtorno; sendo assim quanto mais próximo de zero, melhor a saúde/bem estar e quanto mais próximo de doze, maior o comprometimento na saúde. A aproximação do score seis denota a presença de transtornos psiquiátricos ${ }^{19}$.

As variáveis independentes investigadas foram referentes às características demográficas, socioeconômicas e comportamentais, e às condições normativas e subjetivas de saúde. As variáveis relativas às condições demográficas foram: sexo (feminino, masculino) e idade ( 60 a 67 anos, 68 a 75 anos e 76 anos ou mais). As condições socioeconômicas foram avaliadas por: situação conjugal (com companheiro, sem companheiro), raça autodeclarada (branco, amarelo, negro pardo), escolaridade (sem escolaridade, 1 a 4 anos, 5 a 8 anos e 9 anos ou mais) e renda per capita (1 salário ou mais, menos de 1 salário). No grupo de comportamentos foram avaliados os hábitos tabagista (ausente, presente), etilista (ausente, presente) e pratica de atividade física (ativo, nem ativo/nem inativo, inativo). As condições normativas de saúde avaliadas foram: uso de medicamentos (não, sim) e presença de doença sistêmica (ausência, presença). No que diz respeito às questões subjetivas de saúde - principais variáveis independentes - avaliou-se a satisfação com a vida (muito satisfeito, satisfeito, nem satisfeito, nem insatisfeito, insatisfeito e muito insatisfeito), sendo posteriormente classificados como "satisfeitos" (muito satisfeito, satisfeito, nem satisfeito, nem insatisfeito) e "insatisfeitos" (insatisfeito e muito insatisfeito). A qualidade de vida foi avaliada pela versão validada no Brasil do 12 - Item Short-Form Health Survey (SF-12), a partir de seus domínios físico e mental (com risco, sem risco) cujos pontos de corte nos escores gerados foram 56,57 e 60,75, respectivamente, nos quais a qualidade de vida insatisfatória nos domínios referidos foram estimadas por valores superiores a esses pontos de corte $\mathrm{e}^{20,21}$.

As condições de saúde bucal avaliadas foram divididas em condições normativas e subjetivas. As normativas avaliadas foram: edentulismo (ausente, presente), uso de prótese (ausente, presente), necessidade de prótese total superior (não necessita, necessita) e inferior (não necessita, necessita), necessidade de prótese superior e/ou inferior (não necessita, necessita), índice de placa visível (edêntulo, ausente, presente), sangramento gengival (edêntulo, ausente, presente), cálculo (edêntulo, ausente, presente), bolsa periodontal (edêntulo, ausente, presente), perda de inserção (edêntulo, ausente, presente), condição periodontal comprometida (edêntulo, ausente, presente), alteração de tecido mole (ausente, presente), necessidade de tratamento dentário (edêntulo, ausente, presente), necessidade de tratamento odontológico (ausente, presente). O Índice de Placa Visível - IPV foi utilizado para avaliação normativa da higiene bucal, com ligeira modificação, tendo como referência somente seis dentes índices (11, 31, 16, 26, 36 e 46). A avaliação da condição periodontal utilizou o Índice Periodontal Comunitário (CPI), que permite avaliar a condição periodontal quanto à higidez, sangramento e presença de cálculo dentário ou bolsa periodontal, atual proposta da $\mathrm{OMS}^{14}$, sendo complementada pelo exame da Perda de Inserção Periodontal (PIP). Na avaliação do comprometimento da condição periodontal considerou-se como "presente" os idosos que tiveram pelo menos uma das avaliações das condição periodontal comprometida (sangramento, cálculo, bolsa periodontal e perda de inserção). Na necessidade de tratamento dentário foi considerada a avaliação apenas do elemento dental (nenhum tratamento, restauração de uma superfície, restauração de duas ou mais superfícies, coroa por qualquer razão, faceta estética, tratamento pulpar e restauração, extração, remineralização de mancha branca), de acordo com os parâmetros estabelecidos pela $\mathrm{OMS}^{14}$. Já na análise de trata- 
mento odontológico, considerou-se a avaliação da necessidade de tratamento dentário, tratamento periodontal e de prótese dentária, sendo considerado como "presente" os idosos que necessitavam de tratamento em pelo menos uma das avaliações.

Quanto às condições subjetivas de saúde bucal foi avaliada a autopercepção da saúde bucal (positiva, negativa), da mastigação (positiva, negativa), da aparência de dentes e gengivas (positiva, negativa), da fala devido aos dentes e gengivas (positiva, negativa) do relacionamento com outras pessoas em função da saúde bucal (não afeta, afeta) e da dor de dentes e gengivas nos últimos seis meses (nenhuma dor, alguma dor).

Para a análise dos dados, empregou-se o software SPSS ${ }^{\circledast}$ Statistics 18.0. Na análise descritiva incluiu-se a frequência absoluta (n) e a relativa (\%) para variáveis categóricas. Para as variáveis quantitativas foram apresentadas medidas de média e desvio padrão (DP). Em seguida foram conduzidas análises univariadas a partir do Teste Qui-quadrado. Foram incluídas nas análises múltiplas aquelas variáveis que apresentaram nível de significância (valor-p) igual ou inferior a $0,20^{13,22}$. Foram feitas regressões logísticas para estimativa do modelo múltiplo (OR), ficando retido no modelo os fatores que permaneceram associados ao nível de $\mathrm{p} \leq 0,05$, com intervalos de 95\% de confiança (IC95\%). Os princípios éticos deste estudo estiveram de acordo com a Resolução do Conselho Nacional de Saúde (CNS) do Brasil, n $196 / 96$, sob aprovação do Comitê de Ética em Pesquisa, CEP/Unimontes.

\section{Resultados}

Estimou-se a participação de 461 idosos do município, foram encontrados nos domicílios 459, dos quais 419 participaram ( $\mathrm{TR}=90,80 \%)$. A prevalência dos transtornos mentais comuns foi de $44,6 \%$, referente a 187 idosos. A média de idade dos idosos foi de 71,72 anos (DP $=8,74)$, a escolaridade média de 1,70 anos de estudo (DP $=2,50$ ) e a renda per capita de 353,82 reais (DP $=258,69)$. A maioria dos entrevistados foi do sexo feminino $(58,7 \%)$, se autodeclarou como parda $(53,2 \%)$, possuía alguma doença sistêmica $(87,3 \%)$ e apresentou qualidade de vida satisfatória (Tabela 1).

$\mathrm{Na}$ análise descritiva do QSG constatou-se que a maioria dos idosos respondeu positivamente as questões (Tabela 2).

Quanto à análise univariada constatou-se a associação $(p \leq 0,20)$ entre a presença de trans- tornos mentais comuns com variáveis demográficas e com condições normativas e subjetivas de saúde (Tabela 3).

$\mathrm{Na}$ análise múltipla mantiveram-se associadas $(\mathrm{p} \leq 0,05)$ a presença de transtornos mentais comuns e as seguintes variáveis: sexo, satisfação com a vida, domínio mental e físico da qualidade de vida, autopercepção da aparência de dentes e gengivas e do relacionamento em função da saúde bucal (Tabela 4).

\section{Discussão}

O crescimento da população idosa provoca um aumento de pessoas com risco de adquirir doenças neurológicas e psiquiátricas ${ }^{23}$. Tais doenças constituem um grave problema clínico e de saúde pública, além de associarem-se a um pior padrão de qualidade de vida relativa à saúde ${ }^{24,25}$. Entre os idosos de Ibiaí a prevalência de transtornos mentais comuns foi de $44,6 \%$, bem superior às taxas encontradas em Fortaleza - CE $(26,4 \%)^{6}$, Montes Claros - MG $(29,3 \%)^{7}$, Feira de Santana - BA $(29,9 \%)^{26}$, e na China $(26 \%)^{27}$. Muitos fatores contribuem para tal aumento, como o crescimento do número de morbidades e incapacidades inerentes ao envelhecimento, eventos estressantes da vida, isolamento social e dificuldades econômicas ${ }^{26,28}$. A maior prevalência de transtornos mentais entre os idosos de Ibiaí esteve associada às características sociodemográficas e, principalmente, a condições subjetivas de saúde.

A presença destes transtornos entre os idosos do presente estudo foi menor entre homens, corroborando estudos prévios que haviam identificado menor prevalência desses transtornos no sexo masculino ${ }^{8,26,27}$. Homens e mulheres desempenham papéis distintos na sociedade. A maioria das mulheres atualmente tem dupla jornada de trabalho, exercem funções oriundas da sua inserção no mercado de trabalho formal, assim como referentes ao seu papel familiar, dentre elas as tarefas domésticas e a educação dos filhos. Essa sobrecarga de trabalho as torna mais propensas a situações de conflitos, cansaço, estresse e sofrimento, situação que pode estar associada a uma maior morbidade psíquica nesse grupo. Além disso, as mulheres têm uma maior autopercepção negativa da saúde quando comparadas aos homens, sendo assim, expressam com maior facilidade seus sintomas ${ }^{29,30}$ e reconhecem mais prontamente a presença de doenças.

A presença de transtornos mentais foi maior entre os idosos que relataram insatisfação com a 
Tabela 1. Caracterização da população idosa de Ibiaí/MG/Brasil, 2011/2012, quanto à presença de transtornos mentais, condições demográficas, socioeconômicas, comportamentos, condições normativas e subjetivas de saúde. $\mathrm{n}=419$.

\begin{tabular}{|c|c|c|c|c|c|}
\hline Variáveis & $\mathbf{n}$ & $\%$ & Variáveis & $\mathbf{n}$ & $\%$ \\
\hline Transtornos mentais comuns & & & Condições subjetivas de saúde & & \\
\hline Ausência & 156 & 37,2 & Satisfação com a vida* & & \\
\hline Presença & 263 & 62,8 & Muito satisfeito & 99 & 23,8 \\
\hline Demográficas & & & Satisfeito & 108 & 26,0 \\
\hline Sexo & & & Nem satisfeito, nem insatisfeito & 42 & 10,1 \\
\hline Feminino & 246 & 58,7 & Insatisfeito & 156 & 37,5 \\
\hline Masculino & 173 & 41,3 & Muito insatisfeito & 11 & 2,6 \\
\hline Idade & & & Domínio Mental $^{*}$ & & \\
\hline 60 a 67 anos & 164 & 39,1 & Sem risco & 349 & 84,3 \\
\hline 68 a 75 anos & 130 & 31,0 & Com risco & 65 & 15,7 \\
\hline 76 anos ou mais & 125 & 29,9 & Domínio Físico* & & \\
\hline Socioeconômicas & & & Sem risco & 189 & 45,7 \\
\hline Situação conjugal & & & Com risco & 225 & 54,3 \\
\hline Com companheiro & 210 & 50,1 & Condições normativas de saúde bucal & & \\
\hline Sem companheiro & 209 & 49,9 & Edentulismo* & & \\
\hline Raça autodeclarada & & & Ausente & 145 & 35,0 \\
\hline Branco & 62 & 14,8 & Presente & 269 & 65,0 \\
\hline Amarelo & 6 & 1,4 & Uso de prótese $^{*}$ & & \\
\hline Negro & 128 & 30,5 & Ausente & 187 & 45,2 \\
\hline Pardo & 223 & 53,3 & Presente & 227 & 54,8 \\
\hline Escolaridade $^{*}$ & & & Necessidade de prótese total superior & & \\
\hline Sem escolaridade & 217 & 52,2 & Não necessita & 149 & 36,0 \\
\hline 1 a 4 anos de escolaridade & 165 & 39,7 & Necessita & 265 & 64,0 \\
\hline 5 a 8 anos de escolaridade & 23 & 5,5 & Necessidade de prótese total inferior ${ }^{*}$ & & \\
\hline 9 anos ou mais de escolaridade & 11 & 2,6 & Não necessita & 165 & 39,9 \\
\hline Renda per capita* & & & Necessita & 249 & 60,1 \\
\hline 1 salário ou mais & 98 & 33,3 & Necessidade de prótese superior e ou & & \\
\hline Menos de 1 salário & 196 & 66,7 & inferior ${ }^{*}$ & & \\
\hline Comportamentos & & & Não necessita & 56 & 13,5 \\
\hline Hábito tabagista* & & & Necessita & 358 & 86,5 \\
\hline Ausente & 214 & 51,2 & Placa $^{*}$ & & \\
\hline Presente & 204 & 48,8 & Edêntulo & 269 & 65,8 \\
\hline Hábito etilista* & & & Ausente & 24 & 5,8 \\
\hline Ausente & 236 & 56,5 & Presente & 116 & 28,4 \\
\hline Presente & 182 & 43,5 & Sangramento gengival ${ }^{*}$ & & \\
\hline Prática de atividade física* & & & Edêntulo & 269 & 65,6 \\
\hline Ativo & 99 & 23,7 & Ausente & 46 & 11,2 \\
\hline Nem ativo, nem inativo & 69 & 16,5 & Presente & 95 & 23,2 \\
\hline Inativo & 249 & 59,8 & Cálculo* & & \\
\hline Condições normativas de saúde & & & Edêntulo & 269 & 65,6 \\
\hline Uso de medicamentos ${ }^{*}$ & & & Ausente & 22 & 5,4 \\
\hline Não & 105 & 25,2 & Presente & 119 & 29,0 \\
\hline $\operatorname{Sim}$ & 312 & 74,8 & Bolsa periodontal $^{*}$ & & \\
\hline Presença de doença sistêmica* & & & Edêntulo & 269 & 65,6 \\
\hline Ausente & 53 & 12,7 & Ausente & 79 & 19,3 \\
\hline Presente & 365 & 87,3 & Presente & 62 & 15,1 \\
\hline
\end{tabular}

continua

vida no presente estudo. A satisfação com a vida na velhice pode estar relacionada à sensação de conforto, bem-estar, saúde, relações pessoais, en- tre outros ${ }^{31}$, podendo ser uma avaliação subjetiva da qualidade de vida. Era esperado que os idosos acometidos por TMC relatassem insatisfação 


\begin{tabular}{|c|c|c|}
\hline Variáveis & $\mathbf{n}$ & $\%$ \\
\hline \multicolumn{3}{|l|}{ Perda de inserção* } \\
\hline Edêntulo & 269 & 65,9 \\
\hline Ausente & 29 & 7,1 \\
\hline Presente & 110 & 27,0 \\
\hline \multicolumn{3}{|l|}{$\begin{array}{l}\text { Condição periodontal } \\
\text { comprometida }\end{array}$} \\
\hline Edêntulo & 269 & 66,1 \\
\hline Ausente & 7 & 1,7 \\
\hline Presente & 131 & 32,2 \\
\hline \multicolumn{3}{|c|}{ Alteração de tecido mole ${ }^{*}$} \\
\hline Ausente & 328 & 79,2 \\
\hline Presente & 86 & 20,8 \\
\hline \multicolumn{3}{|c|}{ Necessidade de tratamento dentário* } \\
\hline Edêntulo & 269 & 65,0 \\
\hline Ausente & 26 & 6,3 \\
\hline Presente & 119 & 28,7 \\
\hline \multicolumn{3}{|c|}{$\begin{array}{l}\text { Necessidade de tratamento } \\
\text { odontológico }^{*}\end{array}$} \\
\hline Ausente & 37 & 8,9 \\
\hline Presente & 377 & 91,1 \\
\hline \multicolumn{3}{|c|}{ Condições subjetivas de saúde bucal } \\
\hline \multicolumn{3}{|c|}{ Autopercepção... } \\
\hline \multicolumn{3}{|l|}{...da saúde bucal ${ }^{*}$} \\
\hline Positiva & 393 & 94,7 \\
\hline Negativa & 22 & 5,3 \\
\hline \multicolumn{3}{|l|}{...da mastigação* } \\
\hline Positiva & 369 & 88,9 \\
\hline Negativa & 46 & 11,1 \\
\hline \multicolumn{3}{|c|}{...da aparência de dentes e gengivas ${ }^{*}$} \\
\hline Positiva & 360 & 87,4 \\
\hline Negativa & 52 & 12,6 \\
\hline \multicolumn{3}{|c|}{$\begin{array}{l}\text {...da fala devido aos dentes e } \\
\text { gengivas }^{*}\end{array}$} \\
\hline Positiva & 392 & 95,1 \\
\hline Negativa & 20 & 4,9 \\
\hline \multicolumn{3}{|c|}{$\begin{array}{l}\text {...do relacionamento com outras } \\
\text { pessoa em funcão da saúde bucal }\end{array}$} \\
\hline Não afeta & 293 & 71,1 \\
\hline Afeta & 119 & 28,9 \\
\hline \multicolumn{3}{|c|}{$\begin{array}{l}\text {...dor de dentes e gengivas nos } \\
\text { últimos } 6 \text { meses }^{*}\end{array}$} \\
\hline Nenhuma dor & 346 & 83,4 \\
\hline Alguma dor & 69 & 16,6 \\
\hline
\end{tabular}

*Valores com n menor que os respondentes. ${ }^{* *}$ Salário mínimo em 2010 vigente na época da coleta de dados, início de 2011, de $\mathrm{R} \$ 510,00$ correspondente a $\$ 309,05$ dólares.

com a vida, em função das possíveis consequências desses transtornos.

Em Ibiaí, os idosos com transtorno mental apresentaram maior comprometimento dos domínios físico e mental da qualidade de vida. A qualidade de vida relacionada à saúde, em par- ticular, abrange o impacto da doença e do tratamento na capacidade de uma pessoa para realizar atividades diárias, sendo um conceito multiatributo que engloba diversas dimensões, inclusive física, mental ou funcional e bem-estar psicológico ${ }^{32}$. A prevalência de doenças crônicas e múltiplas, dentre as quais os transtornos mentais comuns, têm aumentado entre idosos, ocasionando na maioria das vezes incapacidades ou limitações funcionais, sociais e psicológicas na vida diária das pessoas, comprometendo assim os domínios físico e mental deles ${ }^{33,34}$. Além disso, ressalta-se a possibilidade de causalidade reversa nessa associação. Condições subjetivas de saúde, ou seja, autopercepção das condições de saúde, também podem estar associadas à saúde mental.

A autopercepção é a interpretação que uma pessoa faz do seu estado de saúde e das suas experiências no contexto de sua vida diária. Em geral, esse julgamento é baseado nas informações e nos conhecimentos disponíveis sobre saúde e doença, pode ainda ser mediado pela experiência prévia e pelo contexto social, cultural e histórico da pessoa $^{35,36}$. Estudos conduzidos entre idosos brasileiros evidenciaram que a autopercepção negativa da saúde bucal foi maior entre idosos dentados do que entre edentados, possivelmente pelo fato dos poucos dentes remanescentes na boca apresentarem-se em condições precárias, agindo como fatores causais de dor; interferências negativas na aparência, mastigação e relacionamentos desses idosos $^{36-38}$. A autoavaliação da saúde bucal é multidimensional refletindo a experiência subjetiva das pessoas sobre o seu bem-estar funcional e social $^{39,40}$. Existem evidências de que a autopercepção negativa da aparência é a principal explicação para autopercepção negativa da saúde bucal ${ }^{36}$. Ressalta-se ainda que idosos com autopercepção e ou condições cognitivas comprometidas podem apresentar piores condições de saúde bucal ${ }^{22,41}$.

Entre idosos de Ibiaí, a autopercepção negativa da aparência dos dentes e gengivas foi maior entre aqueles com transtornos mentais comuns, estudos prévios não avaliaram tal associação. A autoavaliação da saúde bucal pode estar associada a fatores objetivos e subjetivos, portanto, não dissocia-se da saúde em geral ${ }^{36,42}$, pois essa autoavaliação sofre influência da presença de doenças sistêmicas, da saúde mental e ou da condição normativa de saúde bucal. Os idosos de Ibiaí, em sua maioria, autoperceberam a sua saúde bucal de forma positiva, porém a autopercepção negativa da aparência em função da condição de saúde bucal parece interferir na vida dessas pessoas podendo gerar transtornos mentais comuns. Além 
Tabela 2. Distribuição da frequência da população idosa de Ibiaí/MG, quanto aos transtornos mentais comuns, $2011 / 2012, n=419$.

\begin{tabular}{|c|c|c|c|c|c|}
\hline Variáveis & $\mathbf{n}$ & $\%$ & Variáveis & $\mathbf{n}$ & $\%$ \\
\hline $\begin{array}{l}\text { Você tem conseguido se concentrar } \\
\text { bem naquilo que faz?*}\end{array}$ & & & $\begin{array}{l}\text { Você tem realizado com satisfação suas } \\
\text { atividades normais do dia-a-dia? }\end{array}$ & & \\
\hline Muito menos do que o de costume & 25 & 6,0 & Muito menos do que o de costume & 24 & 5,7 \\
\hline Menos do que o de costume & 109 & 26,1 & Menos do que o de costume & 45 & 10,7 \\
\hline O mesmo de sempre & 261 & 62,4 & O mesmo de sempre & 324 & 77,4 \\
\hline Melhor do que o de costume & 23 & 5,5 & Melhor do que o de costume & 26 & 6,2 \\
\hline $\begin{array}{l}\text { Você tem perdido o sono } \\
\text { frequentemente por causa das suas }\end{array}$ & & & $\begin{array}{l}\text { Você tem sido capaz de enfrentar seus } \\
\text { problemas adequadamente? }\end{array}$ & & \\
\hline preocupações? & & & Muito menos do que o de costume & 14 & 3,3 \\
\hline Um pouco mais do que o de costume & 173 & 41,3 & Menos do que o de costume & 94 & 22,4 \\
\hline Não mais do que o de costume & 97 & 23,2 & O mesmo de sempre & 274 & 65,5 \\
\hline Muito menos do que o de costume & 42 & 10,0 & Melhor do que o de costume & 37 & 8,8 \\
\hline De jeito nenhum & 107 & 25,5 & Você tem se sentido infeliz e & & \\
\hline Você tem sentido que está & & & deprimido $(\mathrm{a}) ?^{*}$ & & \\
\hline desempenhando um papel útil na vida? & & & Um pouco mais do que o de costume & 101 & 24,2 \\
\hline Muito menos do que o de costume & 26 & 6,2 & Não mais do que o de costume & 82 & 19,6 \\
\hline Menos do que o de costume & 101 & 24,1 & Muito menos do que o de costume & 36 & 8,6 \\
\hline O mesmo de sempre & 258 & 61,6 & De jeito nenhum & 199 & 47,6 \\
\hline Melhor do que o de costume & 34 & 8,1 & Você tem perdido a confiança em si & & \\
\hline Você tem se sentido capaz de tomar & & & mesmo $(a) ?$ & & \\
\hline decisões? & & & Muito menos do que o de costume & 12 & 2,9 \\
\hline Muito menos do que o de costume & 22 & 5,3 & Menos do que o de costume & 52 & 12,4 \\
\hline Menos do que o de costume & 88 & 21,0 & O mesmo de sempre & 99 & 23,6 \\
\hline O mesmo de sempre & 291 & 69,5 & Melhor do que o de costume & 256 & 61,1 \\
\hline Melhor do que o de costume & 18 & 4,2 & Você tem pensado que é uma pessoa & & \\
\hline Você tem se sentido constantemente & & & inútil? & & \\
\hline esgotado (a) e sob pressão? & & & Muito menos do que o de costume & 7 & 1,7 \\
\hline Um pouco mais do que o de costume & 192 & 45,8 & Menos do que o de costume & 31 & 7,4 \\
\hline Não mais do que o de costume & 75 & 17,9 & O mesmo de sempre & 76 & 18,2 \\
\hline Muito menos do que o de costume & 30 & 7,2 & Melhor do que o de costume & 304 & 72,7 \\
\hline De jeito nenhum & 122 & 29,1 & Você se sente razoavelmente feliz, & & \\
\hline Você tem tido a sensação de que não & & & considerando todas as circunstâncias? & & \\
\hline pode superar suas dificuldades?* & & & Muito menos do que o de costume & 10 & 2,4 \\
\hline Um pouco mais do que o de costume & 98 & 23,4 & Menos do que o de costume & 40 & 9,5 \\
\hline Não mais do que o de costume & 113 & 27,0 & O mesmo de sempre & 281 & 67,1 \\
\hline Muito menos do que o de costume & 50 & 12,0 & Melhor do que o de costume & 88 & 21,0 \\
\hline De jeito nenhum & 157 & 37,6 & & & \\
\hline
\end{tabular}

*Valores com n menor que os respondentes.

disso, precárias condições de saúde bucal, possivelmente, são responsáveis pela autopercepção negativa da saúde bucal das pessoas com transtornos mentais comuns. Considera-se ainda a possibilidade de causalidade reversa nesta associação.

Apesar dos idosos investigados possuírem precárias condições de saúde bucal e a autopercepção negativa da aparência dos dentes e gengivas estar associada à presença de TMC, estas parecem não influenciar nas relações sociais já que a presença de TMC foi menor entre aqueles em que a saúde bucal não afetou o relacionamento com outras pessoas. Ressalta-se que o processo de envelhecimento pode ser acompanhado de outros problemas e comprometimentos sistêmicos ${ }^{2,4}$, sendo que este conjunto de fatores pode impactar de forma mais drástica do que problemas bucais. Além disso, observa-se entre idosos brasileiros uma conformação com suas condiçõoes de saúde bucal, mesmo sendo precarias ${ }^{36}$, o que evidencia o baixo impacto dessas condições na vida diária e no relacionamento com outras pessoas.

Quanto a limitações da investigação é importante salientar que a associação entre transtornos 
Tabela 3. Análise univariada dos fatores associados a transtornos mentais comuns entre idosos. Ibiaí - MG, Brasil, 2011/2012 ( $\mathrm{n}=419)$.

\begin{tabular}{|c|c|c|c|c|c|c|c|}
\hline \multirow{3}{*}{ Variáveis } & \multicolumn{4}{|c|}{ Transtornos mentais comuns } & \multirow{3}{*}{ OR } & \multirow{3}{*}{ IC } & \multirow{3}{*}{$\mathrm{p}$} \\
\hline & \multicolumn{2}{|c|}{ Não } & \multicolumn{2}{|c|}{ Sim } & & & \\
\hline & $\mathbf{n}$ & $\%$ & $\mathbf{n}$ & $\%$ & & & \\
\hline \multicolumn{8}{|l|}{ Demográficas } \\
\hline \multicolumn{8}{|l|}{ Sexo } \\
\hline Feminino & 114 & 46,3 & 132 & 53,7 & 1,00 & & \\
\hline Masculino & 118 & 68,2 & 55 & 31,8 & 0,40 & $0,27-0,6$ & 0,000 \\
\hline \multicolumn{8}{|l|}{ Idade } \\
\hline 67 anos ou menos & 105 & 64 & 59 & 36 & 1,00 & & \\
\hline 68 a 75 anos & 69 & 53,1 & 61 & 46,9 & 1,57 & $0,98-2,52$ & 0,058 \\
\hline 76 anos ou mais & 58 & 46,4 & 67 & 53,6 & 2,06 & $1,28-3,31$ & 0,003 \\
\hline \multicolumn{8}{|l|}{ Socioeconômicas } \\
\hline \multicolumn{8}{|l|}{ Situação conjugal } \\
\hline Com companheiro & 122 & 58,1 & 88 & 41,9 & 1,00 & & \\
\hline Sem companheiro & 110 & 52,6 & 99 & 47,4 & 1,25 & $0,85-1,84$ & 0,261 \\
\hline \multicolumn{8}{|l|}{ Raça dicotomizada } \\
\hline Branco/Amarelo & 34 & 50 & 34 & 50 & 1,00 & & \\
\hline Pardo/Negro/Índio & 198 & 56,4 & 153 & 43,6 & 0,77 & $0,46-1,3$ & 0,331 \\
\hline \multicolumn{8}{|l|}{ Escolaridade ${ }^{*}$} \\
\hline Sem escolaridade & 110 & 50,7 & 107 & 49,3 & 1,00 & & \\
\hline 1 a 4 anos de escolaridade & 98 & 59,4 & 67 & 40,6 & 0,70 & $0,47-1,06$ & 0,091 \\
\hline 5 a 8 anos de escolaridade & 15 & 65,2 & 8 & 34,8 & 0,55 & $0,22-1,35$ & 0,190 \\
\hline 9 anos ou mais de escolaridade & 8 & 72,7 & 3 & 27,3 & 0,39 & $0,1-1,49$ & 0,167 \\
\hline \multicolumn{8}{|l|}{ Renda per capita } \\
\hline 1 salário ou mais & 62 & 63,3 & 36 & 36,7 & 1,00 & & \\
\hline Menos de 1 salário mínimo & 118 & 60,2 & 78 & 39,8 & 1,14 & $0,69-1,88$ & 0,612 \\
\hline \multicolumn{8}{|l|}{ Comportamentos } \\
\hline \multicolumn{8}{|l|}{ Hábito tabagista* } \\
\hline Ausente & 112 & 52,3 & 102 & 47,7 & 1,00 & & \\
\hline Presente & 119 & 58,3 & 85 & 41,7 & 0,78 & $0,53-1,15$ & 0,218 \\
\hline \multicolumn{8}{|l|}{ Hábito etilista* $^{*}$} \\
\hline Ausente & 126 & 53,4 & 110 & 46,6 & 1,00 & & \\
\hline Presente & 105 & 57,7 & 77 & 42,3 & 0,84 & $0,57-1,24$ & 0,38 \\
\hline \multicolumn{8}{|l|}{ Prática de atividade física ${ }^{*}$} \\
\hline Ativo & 64 & 64,6 & 35 & 35,4 & 1,00 & & \\
\hline Nem ativo, nem inativo & 41 & 59,4 & 28 & 40,6 & 1,25 & $0,66-2,35$ & 0,492 \\
\hline Inativo & 126 & 50,6 & 123 & 49,4 & 1,79 & $1,1-2,89$ & 0,018 \\
\hline \multicolumn{8}{|l|}{ Condições normativas de saúde } \\
\hline \multicolumn{8}{|l|}{ Uso de medicamentos } \\
\hline Não & 63 & 60 & 42 & 40 & 1,00 & & \\
\hline Sim & 168 & 53,8 & 144 & 46,2 & 1,29 & $0,82-2,02$ & 0,273 \\
\hline \multicolumn{8}{|l|}{ Presença de doença crônica* } \\
\hline Não & 37 & 69,8 & 16 & 30,2 & 1,00 & & \\
\hline Sim & 194 & 53,2 & 171 & 46,8 & 2,04 & $1,1-3,8$ & 0,025 \\
\hline
\end{tabular}

mentais comuns e condições subjetivas de saúde é dinâmica. Portanto, causas e efeitos variam ao longo da vida em uma relação de retroalimentação, assim, em estudos transversais não é possível estabelecer uma relação temporal entre as asso- ciações observadas. Algumas variáveis que podem confundir tal associação não foram analisadas nesta investigação. Ressalta-se, ainda, que o questionário utilizado tenha limitações a serem consideradas. Por outro lado, acredita-se que os resul- 
Tabela 3. continuação

\begin{tabular}{|c|c|c|c|c|c|c|c|}
\hline \multirow{3}{*}{ Variáveis } & \multicolumn{4}{|c|}{ Transtornos mentais comuns } & \multirow{3}{*}{ OR } & \multirow{3}{*}{ IC } & \multirow{3}{*}{$\mathbf{p}$} \\
\hline & \multicolumn{2}{|c|}{ Não } & \multicolumn{2}{|c|}{ Sim } & & & \\
\hline & $\mathbf{n}$ & $\%$ & $\mathbf{n}$ & $\%$ & & & \\
\hline \multicolumn{8}{|l|}{ Condições subjetivas de saúde } \\
\hline \multicolumn{8}{|l|}{ Satisfação com a vida* } \\
\hline Satisfeito & 151 & 60,6 & 98 & 39,4 & 1,00 & & \\
\hline Insatisfeito & 80 & 47,9 & 87 & 52,1 & 1,68 & $1,13-2,49$ & 0,011 \\
\hline \multicolumn{8}{|c|}{ Qualidade de vida - Domínio Mental ${ }^{*}$} \\
\hline Sem risco & 207 & 59,3 & 142 & 40,7 & 1,00 & & \\
\hline Com risco & 23 & 35,4 & 42 & 64,6 & 2,66 & $1,53-4,62$ & 0,001 \\
\hline \multicolumn{8}{|c|}{ Qualidade de vida - Domínio Físico* } \\
\hline Sem risco & 119 & 63 & 70 & 37 & 1,00 & & \\
\hline Com risco & 111 & 49,3 & 114 & 50,7 & 1,75 & $1,18-2,59$ & 0,006 \\
\hline \multicolumn{8}{|c|}{ Condições normativas de saúde bucal } \\
\hline \multicolumn{8}{|c|}{ Edentulismo* } \\
\hline Ausente & 85 & 58,6 & 60 & 41,4 & 1,00 & & \\
\hline Presente & 144 & 53,5 & 125 & 46,5 & 1,23 & $0,82-1,85$ & 0,321 \\
\hline \multicolumn{8}{|l|}{ Uso de prótese $^{*}$} \\
\hline Não usa & 100 & 53,5 & 87 & 46,5 & 1,00 & & \\
\hline Usa & 129 & 56,8 & 98 & 43,2 & 0,87 & $0,59-1,29$ & 0,495 \\
\hline \multicolumn{8}{|c|}{ Necessidade de tratamento odontológico* } \\
\hline Ausente & 16 & 43,2 & 21 & 56,8 & 1,00 & & \\
\hline Presente & 213 & 56,5 & 164 & 43,5 & 0,59 & $0,3-1,16$ & 0,125 \\
\hline \multicolumn{8}{|c|}{ Condições subjetivas de saúde bucal } \\
\hline \multicolumn{8}{|c|}{ Autopercepção... } \\
\hline \multicolumn{8}{|l|}{...da saúde bucal ${ }^{*}$} \\
\hline Positiva & 220 & 56 & 173 & 44 & 1,00 & & \\
\hline Negativa & 11 & 50 & 11 & 50 & 1,27 & $0,54-3$ & 0,583 \\
\hline \multicolumn{8}{|l|}{...da mastigação* } \\
\hline Positiva & 208 & 56,4 & 161 & 43,6 & 1,00 & & \\
\hline Negativa & 23 & 50 & 23 & 50 & 1,29 & $0,7-2,39$ & 0,413 \\
\hline \multicolumn{8}{|c|}{...da aparência de dentes e gengivas ${ }^{*}$} \\
\hline Positiva & 206 & 57,2 & 154 & 42,8 & 1,00 & & \\
\hline Negativa & 24 & 46,2 & 28 & 53,8 & 1,56 & $0,87-2,8$ & 0,135 \\
\hline \multicolumn{8}{|c|}{...da fala devido aos dentes e gengivas ${ }^{*}$} \\
\hline Positiva & 218 & 55,6 & 174 & 44,4 & 1,00 & & \\
\hline Negativa & 12 & 60 & 8 & 40 & 0,84 & $0,33-2,09$ & 0,700 \\
\hline \multicolumn{8}{|c|}{$\begin{array}{l}\text {...do relacionamento com outras pessoa em } \\
\text { função da saúde bucal }\end{array}$} \\
\hline Não afeta & 155 & 52,9 & 138 & 47,1 & 1,00 & & \\
\hline Afeta & 74 & 62,2 & 45 & 37,8 & 0,68 & $0,44-1,06$ & 0,086 \\
\hline \multicolumn{8}{|c|}{...dor de dentes e gengivas nos últimos 6 meses ${ }^{*}$} \\
\hline Nenhuma dor & 195 & 56,4 & 151 & 43,6 & 1,00 & & \\
\hline Alguma dor & 35 & 50,7 & 34 & 49,3 & 1,25 & $0,75-2,11$ & 0,391 \\
\hline
\end{tabular}

${ }^{*}$ Valor com $\mathrm{n}$ menor que os respondentes

tados apresentados podem ser comuns a idosos de municípios da região que apresentem condições contextuais similares às de Ibiaí, tais como o porte populacional e o índice de desenvolvimento humano dentre outras condições.

\section{Conclusão}

O transtorno mental comum foi identificado na maioria dos idosos investigados, sendo sua presença maior entre idosos do sexo masculino, 
Tabela 4. Análise múltipla entre a presença de transtorno mental leve ajustada por variáveis estaticamente significantes ( $\mathrm{p} \leq 0,05)$, entre idosos de Ibíai/MG, 2011/2012.

\begin{tabular}{|c|c|c|c|}
\hline Variáveis & OR & IC95\% & $\mathbf{p}$ \\
\hline \multicolumn{4}{|l|}{ Demográficas } \\
\hline \multicolumn{4}{|l|}{ Sexo } \\
\hline Feminino & 1,00 & & \multirow{2}{*}{0,003} \\
\hline Masculino & 0,51 & $0,33-0,79$ & \\
\hline \multicolumn{4}{|l|}{ Condições subjetivas de saúde } \\
\hline \multicolumn{4}{|l|}{ Satisfação com a vida } \\
\hline Satisfeito & 1,00 & & \multirow{2}{*}{0,002} \\
\hline Insatisfeito & 2,08 & $1,3-3,34$ & \\
\hline \multicolumn{4}{|l|}{ Domínio Mental da qualidade de vida / Corte $42^{*}$} \\
\hline Sem comprometimento & 1,00 & & \multirow{2}{*}{0,000} \\
\hline Com comprometimento & 4,17 & $1,97-8,84$ & \\
\hline \multicolumn{4}{|l|}{ Domínio Físico da qualidade de vida / Corte $50^{*}$} \\
\hline Sem comprometimento & 1,00 & & \multirow{2}{*}{0,000} \\
\hline Com comprometimento & 2,60 & $1,61-4,17$ & \\
\hline \multicolumn{4}{|l|}{ Condições subjetivas de saúde bucal } \\
\hline \multicolumn{4}{|l|}{ Autopercepção da aparência de dentes e gengivas* } \\
\hline Positiva & 1,00 & & \multirow{2}{*}{0,007} \\
\hline Negativa & 2,93 & $1,34-6,38$ & \\
\hline \multicolumn{4}{|c|}{ Autopercepção do relacionamento com outras pessoas em função da saúde bucal ${ }^{*}$} \\
\hline Não afeta & 1,00 & & \multirow{2}{*}{0,004} \\
\hline Afeta & 0,47 & $0,28-0,79$ & \\
\hline
\end{tabular}

${ }^{*}$ Valores com n menor que os respondentes.

que relataram insatisfação com a vida, com comprometimento dos domínios físico e mental da qualidade de vida, e que possuíam uma autopercepção negativa da aparência dos dentes e gengivas e impacto da saúde bucal no relacionamento com outras pessoas. Dessa forma, identificou-se a associação dos transtornos, principalmente, com piores condições subjetivas de saúde dos idosos, acatando a hipótese em investigação. Portanto, subtende-se que a presença desses transtornos está relacionada a uma pior percepção dos idosos quanto às suas condições de saúde geral e bucal, assim como a qualidade de vida insatisfatória. Ressalta-se ainda que se trata de uma amostra de idosos residentes em um município de pequeno porte populacional que possui indicadores sociais baixos, indicando a possibilidade da influência do ambiente, no qual estão inseridos, na presença desses transtornos e nas questões subjetivas de saúde. Considera-se que as políticas de atenção ao idoso devem considerar a necessidade da assistência a saúde mental dessa população visando garantir um envelhecimento com qualidade de vida e saúde. 


\section{Colaboradores}

AMEBL Martins, JE Nascimento, JGS Souza, MAB Sá, SBL Feres, BP Soares e EF Ferreira participaram igualmente de todas as etapas de elaboração do artigo.

\section{Agradecimentos}

Agradecemos o apoio logístico da Prefeitura Municipal de Ibiaí, da Universidade Estadual de Montes Claros e das Faculdades Unidas do Norte de Minas - FUNORTE, o financiamento da FAPEMIG e a colaboração dos participantes do inquérito. Andréa Maria Eleutério de Barros Lima Martins é bolsista do CNPQ. Ferreira Efigênia Ferreira é bolsista de produtividade do CNPQ.

\section{Referências}

1. Carvalho JAM, Garcia RA. Envelhecimento da população brasileira: um enfoque demográfico. Cad Saude Publica 2003; 19(3):725-733.

2. Christensen K, Doblhammer G, Rau R, Vaupel JW. Ageing populations: the challenges ahead. Lancet 2009; 374:1196-1208.

3. Pereira RJ, Cotta RMM, Franceschin SCC, Ribeiro RCL, Sampaio RF, Priore SE, Cecon PR. Contribuição dos domínios físico, social, psicológico e ambiental para a qualidade de vida global de idosos. Rev Psiquiatr Rio Gd Sul 2006; 28(1):27-38.

4. Calkins E, Ford AB, Katz PR. Geriatria Prática. Rio de Janeiro: Revinter; 1992.

5. Organização Mundial de Saúde (OMS), Organização Pan-Americana de Saúde (OPAS). Relatório sobre a Saúde no mundo 2001: Saúde mental: nova concepção, nova esperança. Geneva: OMS; 2001.

6. Coelho Filho JM, Ramos LR. Epidemiologia do envelhecimento no Nordeste do Brasil: resultados de inquérito domiciliar. Rev Saude Publica 1999; 33(5):445-453.

7. Maia LC, Durante AMG, Ramos L R. Prevalência de transtornos mentais em área urbana no norte de Minas Gerais, Brasil. Rev Saude Publica 2004; 38(5):650-656

8. Borim FSA, Barros MBA, Botega NJ. Transtorno mental comum na população idosa: pesquisa de base populacional no Município de Campinas, São Paulo, Brasil. Cad Saude Publica 2013; 29(7):1415-1426.

9. Goldberg D. The overlap between the common mental disorders - Challenges for classification. Int Rev Psychiatry 2012; 24(6):549-555.

10. Goldberg D, Huxley P. Commom mental disorders: a bio-social model. London: Tavistock; 1992.

11. Velasco-Ortega E, Segura-Egea JJ, Córdoba-Arenas S, Jiménez-Guerra A, Monsalve-Guil L, López-López J. Comparison of the dental status and treatment needs of older adults with and without chronic mental illness in Sevilla, Spain. Med Oral Patol Oral Cir Bucal 2013; 18 (1):71-75.

12. Ekbäck G, Astrøm AN, Klock K, Ordell S, Unell L. Variation in subjective oral health indicators of 65-yearolds in Norway and Sweden. Acta Odontol Scand 2009; 67(4):222-232.

13. Martins AMEBL, Jones KM, Souza JGS, Pordeus IA. Associação entre impactos funcionais e psicossociais das desordens bucais e qualidade de vida entre idosos. Cien Saude Colet 2014; 19(8):3461-3478.

14. World Health Organization (WHO). Oral health surveys: basic methods. $4^{\text {th }}$ ed. Geneva: ORH/EPID; 1997.

15. Fleiss JL. Statistical methods for rates and proportions. New York: John Wiley Sons; 1981.

16. Cicchetti DV, Volkmar F, Sparrow SS, Cohen D, Fermanian J, Rourke BP. Assessing the reliability of clinical scales when the data have both nominal and ordinal features: proposed guidelines for europsychological assessments. J Clin Exp Neuropsychol 1992; 14(5):673686.

17. Goldberg DP. The detection of psychiatric illness by questionnaire. London: Oxford University Press; 1972.

18. Pasquali L, Gouveia VV, Andriola WB, Miranda FJ, Ramos ALM. Questionário de Saúde Geral de Goldberg (QSG): Adaptação brasileira. Psic: Teor e Pesq 1994; $10(3): 421-438$ 
19. Goldberg DP, Jackson G, Gater R, Campbell M, Jennett $\mathrm{N}$. The treatment of common mental disorders by a community team based in primary care: a cost effectiveness study. Psychol Med 1996; 26(3):487-492.

20. Camelier AA. Avaliação da Qualidade de Vida Relacionada à Saúde em Pacientes com DPOC: estudo de base populacional com o SF-12 na cidade de São Paulo-SP [tese]. São Paulo: Universidade Federal de São Paulo; 2004.

21. Sanda MG, Wei JT, Litwin MS. Scoring Instructions for the Expanded Prostate cancer Index Composite (EPIC). EPIC [serial on the Internet] 2002 [cited 2014 Jun 5]. [about 5 p.]. Available from:http://www.med.umich. edu/urology/research/EPIC/EPIC-Scoring-2.2002.pdf

22. Teles MA, Barbosa MR, Vargas AM, Gomes VE, Ferreira EF, Martins AMEBL, Ferreira RC. Psychosocial work conditions and quality of life among primary health care employees: a cross sectional study. Health and Quality of Life Outcomes 2014; 12:72.

23. Prince MJ, Harwood R, Blizard RA, Thomas A, Mann AH. Social support deficits, loneliness and life events as risk factors for depression in old age. The Gospel oak project VI. Psychological Med 1997; 27(2):323-332.

24. Addonizio G, Alexopaulos GS. Affective disorders in the elderly. Int J Geriatr Psychiatric 1993; 8:41-47

25. Jansen K, Modin TC, Ores LC, Souza LDM, Konradt CE, Pinheiro RT, Silva RA. Transtornos mentais comuns e qualidade de vida em jovens: uma amostra populacional de Pelotas, Rio Grande do Sul, Brasil. Cad Saude Publica 2011;27(3):440-448.

26. Rocha SV, Almeida MMG, Araujo TM, Virtuoso Junior JS. Prevalência de transtornos mentais comuns entre residentes em áreas urbanas de Feira de Santana, Bahia. Rev Bras Epidemiol 2010; 13(4):630-640.

27. Liu CY. Depressive disorders among older residents in a Chinese rural community. Psychol Med 1997; 27(4):943.

28. Lima MS, Soares BGO, Mari JJ. Saúde e doença mental em Pelotas, RS: dados de um estudo populacional. Rev Psiq Clín 1999; 26:225-235.

29. Jenkins R. Sex difference in minor psychiatric morbidity. Cambridge: Cambridge University Press; 1985.

30. Brito JC. Enfoque de gênero e relação saúde/trabalho no contexto de reestruturação produtiva e precarização do trabalho. Cad Saude Publica 2000; 16(1):195-204.

31. Joia LC, Ruiz T, Donalisio MR. Life satisfaction among elderly population in the city of Botucatu, Southern Brazil. Rev Saude Publica 2007; 41(1):131-138.
32. Kandelman D, Petersen PE, Ueda H. Oral health, general health, and quality of life in older people. Spec Care Dentist 2008; 28(6):224-236.

33. Duarte MB, Rego MAV. Comorbidade entre depressão e doenças clínicas em um ambulatório de geriatria. $\mathrm{Cad}$ Saude Publica 2007; 23(3):691-700.

34. Boing AF, Melo GR, Boing AC, Moretti-Pires RO, Peres KG, Peres MA. Association between depression and chronic diseases: results from a population-based study. Rev Saude Publica 2012; 46(4):617-623.

35. Gilbert L. Social factors and self-assessed oral health in South Africa. Community Dent Oral Epidemiol 1994; 22(1):47-51.

36. Martins AMEBL, Barreto SM, Pordeus IA. Auto-avaliação de saúde bucal em idosos: análise com base em modelo multidimensional. Cad Saude Pública 2009; 25(2):421-435.

37. Matthias RE, Atchison KA, Lubben JE, De-Jong F, Schweitzer SO. Factors affecting self-ratings of oral health. J Public Health Dent 1995; 55(4):197-204.

38. Bulgarelli AF, Manço ARX. Idosos vivendo na comunidade e a satisfação com a própria saúde bucal. Cien Saude Colet 2008; 13(4):1165-1174.

39. Locker D. Clinical correlates of change in self perceived oral health in older adults. Community Dent Oral Epidemiol 1997; 25(3):199-203.

40. Reis SCGB, Marcelo VC. Saúde bucal na velhice: percepção dos idosos, Goiânia, 2005. Cien Saude Colet 2006; 11(1):191-199.

41. Ferreira RC, Vargas AMD, Fernandes NCN, Souza JGS, Sá MAB, Oliveira LFB, Martins AMEBL. O idoso com comprometimento cognitivo apresenta pior condição de saúde bucal? Cien Saude Colet 2014; 19(8):34173428.

42. Benyamini Y, Leventhal H, Leventahal EA. Self rated oral health as an independent predictor of self rated general health, self esteem and life satisfaction. Soc Sci Med 2004; 59(5):1109-1116.

Artigo apresentado em 29/07/2014

Aprovado em 01/10/2015

Versão final apresentada em 03/10/2015 